\title{
Competencias personales y gerenciales de los Directores y Subdirectores de las Escuelas Básicas
}

\author{
Marcano, Noraida* \\ Finol de Franco, Mineira**
}

\section{Resumen}

En esta investigación se determinan las competencias personales y gerenciales que poseen los directores y subdirectores del nivel de educación básica, específicamente, de las escuelas de primera y segunda etapa ubicadas en el Municipio Maracaibo del Estado Zulia. El estudio de tipo descriptivo, de campo, se sustentó teóricamente en Mercader (1998), Quinn (1995), Ruiz (1992), Requeijo y Lugo (1997), entre otros. Se aplicó un cuestionario contentivo de sesenta y cuatro (64) ítems dirigido a 28 directivos que laboran en diez (10) escuelas básicas. El mismo fue validado por ocho (8) expertos y su confiabilidad de 0.88 , calculada mediante el coeficiente de Alpha Cronbach. Los resultados evidencian que, según la percepción de los gerentes educativos encuestados, ellos poseen las competencias personales en un alto dominio de las mismas, específicamente el conocimiento de sí mismo, autorrealización y autocontrol. Igualmente para las competencias gerenciales en los roles de director, mentor y facilitador con promedios por indicador que oscilan desde 2.98 hasta 3.44 ubicándolos en la categoría de alto dominio de la competencia; sin embargo, para el rol de innovador con los indicadores convivir con el cambio (1.66) y pensamiento creativo (1.77) se ubican en muy bajo dominio. Sobre este último rol deberán reflexionar quienes gerencian las escuelas en estudio, dada la importancia del mismo en el marco del proceso de reformas e innovaciones que vive el sistema educativo venezolano.

Palabras clave: Gerente educativo, escuelas básicas, competencias personales, competencias gerenciales.

Recibido: 10-11-05. Aceptado: 28-05-07

* $\quad$ Dra. en Ciencias de la Educación, Profesora Titular de la Universidad del Zulia.

E-mail: noraespinoza@hotmail.com

** Lic. en Educación. Mención Ciencias Sociales. Profesora Titular de la Universidad del Zulia. Doctora en Ciencias de la Educación. E-mail: mineiradefranco@hotmail.com 


\title{
Personal and Managerial Competence in Directors and Sub-Directors of Basic Schools
}

\begin{abstract}
The main objective of this paper was to determine the personal and managerial competences of directors and sub-directors in the first and second stages of basic education, in the Municipality of Maracaibo, State of Zulia. This descriptive-field research was supported theoretically by the work of Mercader (1998), Quinn (1995), Ruiz (1992), Requeijo and Lugo (1997), among others. The questionnaire used consisted of sixty-four (64) items and was administered to twenty-eight (28) directors working at ten (10) elementary schools. This instrument was validated by eight experts and its reliability, calculated using the Cronbach Alpha Coefficient, was 0.88 . Results obtained indicated that the educational directors surveyed have a high mastery level for personal competences, specifically those related to self-knowledge, self-achievement and self-control. Likewise, for managerial competences in the roles of director, advisor and facilitator, results by indicator showed averages oscillating between 2.98 and 3.44 , placing them in the category of a high mastery level for these competences. Nevertheless, regarding the role of innovator for the indicators living with change (1.66) and creative thought (1.77), they show a very low mastery level. School managers should reflect on this last role, given its importance in the process of reform and innovation currently being experienced in the Venezuelan educational system.
\end{abstract}

Key words: Educational manager, basic schools, personal competencies, managerial competencies.

\section{Introducción}

En virtud del papel que debe cumplir la educación en el siglo XXI, el Estado, en el marco de la transformación del sistema educativo, ha venido planteando la reforma del nivel de educación básica, en cuyo campo algunos de los aspectos mas debatidos son el de la formación y desarrollo de competencias.

En este contexto, el desempeño del personal directivo constituye uno de los principales factores de calidad del servicio educativo. De ahí la importancia de contar con directores y subdirectores cuyo perfil integre competencias personales y profesionales como patrones generales de comportamiento y ejecución de acciones concretas, que propicien el desarrollo con excelencia del ejercicio profesional y, de donde emerjan un conjunto de factores, componentes y relaciones entre los distintos actores, que le impriman calidad al sistema educativo.

Lo anterior conduce a plantear que, los directores y subdirectores deben realizar una acción reflexiva que los conduzca a desarrollar una tarea consigo mismo mediante la cual puedan acrecentar el conocimiento y control sobre su dinámica pedagógica; interactuar de manera armónica con el entorno institucional y con el contexto sociocultural generando conocimiento, ejerciendo autonomía, practicando la cooperación y desarrollando lazos de afecto con sus colegas y demás miembros de la comunidad educativa; tomar decisiones, trabajando de manera con- 
junta hacia la consecución, con calidad, de los objetivos institucionales y mejorar su desempeño.

En consecuencia, el papel del docente directivo ha de ser cultural-profesional proyectándose más allá de todas las cualidades que son reconocidas como deseables para legitimar su quehacer y responder a las necesidades sociales e históricas de la comunidad con la que trabaja, para liderar los procesos tanto pedagógicos como administrativos de su escuela, de manera que, mediante una real participación de los distintos actores de la comunidad educativa, y según sus condiciones, se responda a las necesidades reales de las instituciones educativas; porque el personal directivo no apunta solamente al cumplimiento de una política determinada o a la solución de determinados problemas. Antes bien, debe posibilitar la comprensión de los aspectos positivos y negativos surgidos de las interacciones entre los sujetos que intervienen en la educación, ya que la búsqueda da sentido a lo educativo, se estructura mediante el juego de intersubjetividades que hacen explicitas las intencionalidades, las concepciones y enfoques que pretenden ponerse al servicio de la escuela y de la educación; por lo tanto, requiere de acciones de interlocución que no involucren solamente al maestro, sino también, y fundamentalmente, al personal directivo, quienes deben estar en capacidad de ofrecerse para el otro y para sí mismos con el propósito de conducir con éxito e imprimir calidad a las organizaciones educativas que dirigen.

Las reflexiones anteriores orientan esta investigación, cuyo propósito es determinar las competencias personales y profesionales que poseen los directores y subdirectores de la I y II etapas del nivel de Educación Básica, quienes por su investidura como personal directivo de las instituciones educativas deben liderar los procesos pedagógicos y administrativos que se suceden en esas escuelas, propiciando la participación y el compromiso de los distintos actores que hacen vida en esas organizaciones.

\section{Descripción de la situación objeto de estudio}

La educación ha sido considerada como el medio mas importante y el eje fundamental para la transformación y construcción del país "que queremos y necesitamos", por cuanto a través de ella se forma el talento humano y se desarrolla el potencial creativo de las personas para el pleno ejercicio de su personalidad en una sociedad democrática y participativa.

El logro de esta misión asignada a la educación en estos tiempos de globalización económica, política y sociocultural, hacen perentoria la participación y asunción de la corresponsabilidad de todos los actores sociales involucrados en la práctica académica y administrativa que le compete tanto a los intereses de los centros educativos como a la comunidad donde están ancladas. En este sentido, le corresponde a quienes dirigen las instituciones educativas (director y subdirector) garantizar ante la sociedad el cumplimiento a cabalidad de las políticas, objetivos y metas trazados por el Estado venezolano a través del Ministerio de educación y Deportes.

En consecuencia, la calidad educativa depende, en gran parte, de la geren- 
cia del director y subdirector, quienes deben poseer un conjunto de competencias personales, profesionales y gerenciales que les permitan dinamizar las escuelas en pro de alcanzar los objetivos y metas. Particularmente Silíceo (1997) afirma que, el director general de una empresa u organización, independientemente del servicio que preste, debe ser un agente de cambio, un auténtico líder, capaz de movilizar su energía, generando una actitud y un espíritu productivo.

Además, expresa Álvarez (1999) la necesidad de que el director conozca y aplique con sensatez y flexibilidad las normas de funcionamiento y dinámicas de grupo, técnicas de organización, creación de estructuras, reuniones, conducción de equipos, toma de decisiones, manejo de conflictos, planificación, ejecución y control; es decir, que cuente con los conocimientos, habilidades y destrezas inherentes a los roles que debe cumplir; todo ello, para lograr efectividad en la gestión y superar los obstáculos gerenciales y organizativos, que según describen el Ministerio de Educación y Deportes (2003) y el Proyecto Educativo Nacional (2000), existen en las zonas educativas y municipios escolares. Esos obstáculos son:

- Verticalismo y centralismo en las decisiones.

- Rigidez administrativa, excesivos trámites y recaudos

- Planificación normativa, de simple cumplimiento

- Estadísticas poco confiables

- Escasa supervisión y, cuando existe, es básicamente de carácter fiscalizadora y controladora
- Poca comunicación entre instituciones educativas, los municipios escolares y las zonas educativas.

Para superarlos, el Ministerio de Educación y Deportes (2005) propone que, la gerencia dentro de los centros educativos debe propiciar la participación social en el proceso de toma de decisiones, integrar de los diferentes aspectos que conforman la elaboración de proyectos educativos y promocionar un liderazgo transformacional para consolidar espacios legítimos de intercambio de experiencias y libertad de pensamiento.

Todo lo anterior, presupone una concepción de gestión participativa que integre las demandas administrativas, pedagógicas y comunitarias, sobre todo, en las escuelas básicas de primera y segunda etapa, donde el Currículo Básico Nacional (1996) demanda un aprendizaje constructivista, basado en experiencias significativas de los niños y niñas, de participación de todos los actores inmersos en el hecho educativo a través de la elaboración y puesta en marcha de proyectos educativos integrales comunitarios (PEIC) y proyectos pedagógicos de aula (PPA).

En este escenario, los directores y subdirectores de los centros escolares deben ser individuos poseedores de ciertas cualidades personales, capaces de administrar la educación, apoyados en principios y decisiones que normativamente están declarados. Al respecto, el Ministerio de Educación, Cultura y Deportes (2003) destaca que, el director debe desempeñar funciones pedagógicas y de gestión administrativa, mediante un mo- 
delo de gerencia participativa, bajo relación de igualdad y calidad. Unido a esto, se le exige poseer una serie de competencias gerenciales en sus diferentes roles para posibilitar el cumplimiento de una gestión académica y administrativa participativa, centrada en promover y facilitar el aprendizaje, con conciencia social.

En función de los planteamientos anteriores, esta investigación se orientó a determinar y caracterizar las competencias personales y gerenciales que posee el gerente educativo de las escuelas de primera y segunda etapa de educación básica ubicadas en el Municipio Maracaibo.

La investigación se justifica en función de lo expresado por el Ministerio de Educación, Cultura y Deportes (2003), hoy Ministerio Educación y Deportes cuando propone crear condiciones al interior de las escuelas básicas para una gerencia que trascienda las exigencias estrictamente administrativas, atendiendo al componente personal del director y subdirector para que se constituyan en auténticos agentes de cambio desde los centros educativos.

Por otra parte, el nivel de educación básica en la primera y segunda etapa, requiere de talento humano preparado para cumplir con los objetivos y metas establecidos en el Currículo Básico Nacional; por lo tanto, el gerente educativo debe responder a los nuevos enfoques gerenciales demostrando dominio de las competencias básicas y específicas para el desempeño de los roles de director, coordinador, facilitador, mentor e innovador, las cuales se manifestarán en el incremento de los niveles de calidad de las escuelas básicas.

\section{El deber ser del gerente educativo}

El término gerencia, de acuerdo con Ruiz (1992), se refiere a las organizaciones que efectúan actividades de planificación, organización, dirección y control a objeto de utilizar sus recursos humanos, físicos y financieros con la finalidad de alcanzar determinados objetivos; por su parte, Bonilla (2001) expresa que, "la gerencia es un proceso interactivo que otorga al individuo y a los grupos la responsabilidad de crecer y desarrollarse en un ambiente organizacional apto para el aprendizaje".

En este marco de definiciones, cabe señalar que la gerencia educativa, en las escuelas básicas constituye una tarea compleja para el director y subdirector; quienes deben tener claro cuales son sus roles, funciones y tareas a cumplir en las instituciones que dirigen con la finalidad de lograr los objetivos y metas propuestas. La gerencia es entonces, a juicio de Requeijo y Lugo (1997):

La actuación a través de la cual se logra conjugar personas, distribuir tares y repensar sus trabajos a objeto de que el conjunto produzca bien. Es controlar y manejar los recursos materiales y humanos, para ponerlos al servicio de los grandes fines y aspiraciones determinados por la política educacional (p.19).

En este sentido, el gerente educativo como responsable directo y cuentadante del Ministerio de Educación, supervisor nato del centro educativo, debe poseer ciertas competencias personales y profesionales que le permitan conducir y gestionar la organización educativa; debe ser, además, un individuo poseedor 
de un conjunto de competencias personales, profesionales y gerenciales para propiciar un espacio de aprendizaje basado en la participación del equipo de trabajo bajo su dirección.

La gestión del personal directivo debe estar sustentada en estrategias que humanicen sus acciones, haciendo uso de la racionalidad y de procedimientos sistemáticos que vayan desde la planificación hasta el control y seguimiento de las acciones que emprende.

\section{Algunas consideraciones en torno a las competencias}

El término competencias ha sido definido de diversas maneras en correspondencia con la apreciación o punto de vista que tienen los autores en relación a éste. Así, según Boyatzis (citado por Dalziel y otros, 1996) la competencia puede definirse como una "característica subyacente en una persona, que está causalmente relacionada con una actuación exitosa en un puesto de trabajo". En cuanto que, para Dalziel y otros (1996), están condicionadas por motivaciones personales, rasgos de carácter y la imagen que tiene la persona de sí mismo. Carrasco (2003) las define como las evidencias observables en las personas; mientras que para Quinn (1995) significa "poseer los conocimientos y las destrezas necesarias para desempeñar determinadas tareas o rol".

\subsection{Tipos de competencias}

En correspondencia con las definiciones presentadas, igualmente se iden- tifican diferentes tipos de competencias. Para Mercader (1998) las competencias del director están asociadas a los componentes personales; los cuales se traducen en actitudes a desarrollar por el gerente a partir de las competencias que caracterizan su gestión, Además de estas competencias personales, Quinn (1995) establece, entre las competencias gerenciales que debe poseer y aplicar el directivo de una empresa u organización, cinco roles fundamentales, éstos son: Rol de director, coordinador, facilitador, mentor e innovador a cada uno de estos roles igualmente le define ciertas competencias específicas.

Competencias personales del gerente educativo:

La gestión del gerente educativo en las escuelas básicas se debe apoyar en una serie de características o cualidades personales que inciden a su vez en la conducción de la organización que dirige. En efecto, Mercader (1998) expresa que:

El gerente moderno está saturado de libros y cursos de alta gerencia de todo tipo, pero dicho gerente es una persona que tienen numerosas actividades y vivencias totalmente ajenas y separadas de su vida de trabajo, que pueden ser tan importantes o más que ella, según los casos y cada persona.

Entre las competencias personales el autor arriba citado, distingue las siguientes competencias asociadas: conocimiento de sí mismo, autorrealización y autocontrol.

\section{- Conocimiento de sí mismo}

El conocimiento de sí mismo se vincula con la necesidad de que el gerente 
se conozca, que tenga claramente definidos sus deseos e intereses, percepciones y motivaciones.

Desarrollar el conocimiento de sí mismo implica adentrarse en el ámbito de las aspiraciones puestas de manifiesto en los objetivos personales que se posea, es lo que Senge (1997) identifica como "visión personal; es decir, metas intrínsecas relevantes que brotan de un plano más sólido que los propósitos, los cuales por lo general expresan quererse liberar de algo". La visión, explica el autor, es un destino específico, la imagen de un futuro deseado, la visión es concreta. De igual manera, Mercader (1998) explica que, las metas son de carácter psíquico, espiritual o material y, en general, aún sin concientizarlo, se logra la combinación de las tres".

En consecuencia, todo gerente debe establecer prioridades, definir metas, identificar una visión personal y profesional; para ello debe autoevaluarse, ésta le aporta un conocimiento claro de sus aspiraciones, pero también de sus limitaciones. De tal manera que, el conocimiento de sí mismo se fundamenta en la autoevaluación, ésta se convierte en la estrategia por excelencia para detectar las debilidades y fortalezas que poseen y su relación con las oportunidades y amenazas como factores externos que pudieran impedir el logro de sus metas.

\section{- Autorrealización}

Otra de las competencias personales que debe poseer el gerente educativo es la autorrealización, ésta es definida por Senge (1997) como una necesidad fundamental en la adquisición de los propios ideales; es decir, hacer realidad las visiones. Por su parte, Acosta (1998) con- sidera que la autorrealización es "la aspiración que sentimos para ser mejores, por buscar la excelencia en lo que se realice, Es una aspiración que nos motiva. Depende de nuestra voluntad. Es la búsqueda permanente de nuestro propio crecimiento y desarrollo personal".

Vista de esta manera, la autorrealización es consecuencia de la motivación que siente el gerente educativo frente a las visiones que posee. Esta motivación lo impulsa a actuar para el logro del éxito, apoyándose en su fuerza de voluntad y en la confianza en sus capacidades.

Según Mercader (1998) "todos poseemos la capacidad para pensar con claridad, de mostrar decisión, de tener una mentalidad única que permite discernir entre las posibilidades reales, eligiendo la mejor alternativa para la solución de problemas". La autorrealización, según este autor, no sólo abarca alcanzar metas, sino sentir satisfacción por el crecimiento que se experimenta; descubrir que se evoluciona en atención a las posibilidades y destrezas.

\section{- Autocontrol}

El autocontrol enfatiza en la capacidad que debe tener cualquier persona y, entre ellas el gerente educativo del manejo equilibrado de su inteligencia emocional. Permite además al gerente, unificar las aspiraciones que posee en cada rol que desempeña. Mercader (1998) destaca "la presión del trabajo genera cierto nivel del saber para dosificarla. De esta forma se puede llegar a niveles controlables, los cuales logran resultados parciales que en sumatoria son los resultados de vida".

En consecuencia, el campo de acción del autocontrol, se inicia con la res- 
puesta emitida ante toda forma de resistencia que se oponga al logro de metas. El autor antes citado plantea que, es necesario y trascendental para el gerente, educar su mente; la educación de la mente se fundamenta en el cultivo y desarrollo de ciertos hábitos que clarifican el sentido de la vida y el rol en ella. Estos hábitos son: sonreír, aprovechar la vida a plenitud en tiempo agradable, dejarse influenciar por las ideas positivas. El autocontrol emocional permite al gerente controlar los pensamientos, sensaciones y sentimientos.

Las competencias del gerente educativo en su componente personal a través del conocimiento de sí mismo, autorrealización y autocontrol, hacen posible el desarrollo de habilidades y capacidades para actuar teniendo presente los retos que debe enfrentar desde su posición como gerente, más las características o cualidades individuales dentro del componente humano que también está llamado a manejarlo equilibradamente.

\section{Competencias gerenciales:}

Junto a las competencias personales, el personal directivo también debe poseer un conjunto de conocimientos, habilidades y destrezas de tipo gerencial que lo lleven a gestionar eficientemente los centros educativos. En este sentido, diversos autores definen las competencias gerenciales de la siguiente manera:

Quinn (1995) afirma que éstas contribuyen al conjunto de conocimientos, habilidades y destrezas que debe poseer el personal directivo de una empresa para convertirse en verdaderos líderes.

Dalziel y otros (1996) explican, que éstas comprenden la capacidad de emprender acciones eficaces para mejorar el talento y las capacidades de los demás; la capacidad para comunicar lo que es necesario hacer y lograr que cumplan los deseos de uno, teniendo en mente el bien de la organización a largo plazo.

Por su parte, Vásquez (2005) señala que las competencias gerenciales deben reflejar la conducta que se necesita para el futuro éxito de la empresa u organización. Pero éstas deben ser acompañadas por otros factores clave como son la misión, la información y la cultura. Todo ello, como una vía para lograr que la organización tenga los directores que puedan desempeñar un liderazgo apropiado, que los empleados o subordinados sepan qué deben hacer con toda la información que reciben y las competencias que se necesitan se adquieran y estén garantizadas de un modo estructurado.

En este orden de ideas cabe identificar, siguiendo los planteamientos de Quinn (1995), los roles competitivos que debe poseer el directivo o gerente de una empresa u organización. Junto a esos roles, el autor asocia una serie de competencias específicas. Estos roles y competencias se describen a continuación.

1. Rol de director: En este rol se espera que el director clarifique las expectativas personales y las de su equipo, utilizando procesos como la planificación y la fijación de metas, que sea un iniciador decidido, defina los problemas, establezca los objetivos, especifique los roles y tareas, genere las normas y políticas.

Para este rol, Quinn (1995) identifica tres competencias específicas: toma de iniciativa, fijación de metas y delegación eficaz. Estas tres competencias son básicamente similares; pero utilizadas conjuntamente constituyen las activida- 
des básicas de la gestión y la organización, fijan una orientación y una visión clara, desafiante y realista, dan poder y autoridad responsable a los miembros de la organización y les capacitan para que puedan convertir en realidad esa visión.

- Toma de iniciativas: es algo similar a asumir el mando y como director con frecuencia tiene que hacerlo. Para tomar iniciativas Quinn (1995) plantea seguir cinco pasos básicos, estos son: utilizar la motivación interior; centrarse en las tareas más importantes; ser persistente y paciente; ser un pigmalión positivo: contagiar el deseo de la excelencia; catalizar la acción inteligente.

- Fijación de metas: La fijación de metas personales tiene lugar en todos los niveles de la organización independientemente de su razón de ser, sin embargo, aclara Quinn (1995) que la orientación, el propósito y los tipos de actividades que forman parte del proceso varían en correspondencia con los diferentes niveles organizativos y de acuerdo a la naturaleza de la empresa.

Ahora bien ¿Cómo fijar metas personales y organizacionales?; al respecto, se recomienda lo siguiente:

- Seleccionar una meta para su vida académica, laboral o personal y desarrollar un plan de ejecución. Debe incluir: objetivos inteligentes, factores externos que puedan afectarlos y los pasos necesarios para lograrlos y fecha en que lo lograrán.

- Evaluar los resultados posibles.

- Incluir cualquier comentario o explicación sobre un plan.
- Delegación eficaz: Señala Quinn (1995) que, con frecuencia la delegación se confunde con el simple proceso de asignar tareas a los subordinados; sin embargo, significa mucho más pues no solo genera beneficios importantes para el directivo por cuanto le deja mas tiempo libre permitiendo así que centre su atención en temas más significativos. También contribuye a la formación y desarrollo de los subordinados y a la asignación sabia de los recursos organizativos.

Según el autor antes citado, para una delegación eficaz es necesario: clarificar en la mente qué es lo que desea que se haga, escribirlo puede ayudarlo; asignar la tarea deseada al empleado más adecuado; mantener abiertos los canales de comunicación; permitir que los empleados hagan la tarea de la forma que se sientan más cómodos; comprobar el progreso; responsabilizar a la persona del trabajo y de cualquier dificultad que pueda surgir; no ignorar los esfuerzos de los empleados porque puede ser desastroso para la motivación; reconocer los logros y demostrar que los valora adecuadamente.

2. Rol de coordinador: En este rol, la tarea del directivo como gerente educativo, consiste en asegurar que el trabajo fluya adecuadamente y que las diversas actividades se realicen con la importancia del caso, y por supuesto, en una dinámica donde debe asegurarse el mínimo de conflicto entre las personas, los grupos y los diferentes departamentos que conforman los centros educativos.

En el rol de coordinador igualmente, Quinn (1995) señala tres competen- 
cias específicas: planificación, organización y control.

- Planificación: la planificación y la fijación de metas son herramientas importantes para determinar hacia donde se desea ir y cómo desea llegar allí; concebida de esta manera, el autor antes citado identifica tres tipos de planificación: la planificación estratégica o planificación orientada a la fijación de la misión de la organización, la planificación táctica que incluye la ejecución del plan estratégico y la fijación de objetivos específicos para cada departamento y para cada empleado, en éste se especifica cómo se lograrán cada uno de los objetivos, y un tercer tipo de planificación, la llamada planificación operativa que implica decidir cómo se utilizarán los recursos financieros, humanos y materiales para garantizar la entrega de los servicios con la mayor eficacia posible.

- Organización: esta competencia específica asociada al rol de coordinador del gerente educativo, refiere el proceso de dividir el trabajo en componentes manejables y de asignar las actividades para lograr con la mayor eficacia posible los resultados deseados. Acota Quinn (1995) que, si bien la planificación ofrece las herramientas para decidir dónde ir y cuál es la mejor forma de llegar allí, la organización se convierte en las herramientas para realmente llegar allí.

Requeijo y Lugo (1997) explican que, la organización permite diseñar la estructura organizativa de las escuelas; ésta debe tener correspondencia con los cargos existentes y atender los cinco principios de la organización planteados por Ruiz (1992), estos son: especialización, división del trabajo, unidad de mando, cadena jerárquica y control.

- Control: Es un proceso que le indica a quienes gerencian, si realmente se llegó al cumplimiento de los objetivos y metas previamente establecidos a través de la planificación. En este sentido, Quinn (1995) afirma que, para mantener la continuidad y estabilidad de la organización, el gerente utiliza el control como un mecanismo que ofrece información de retorno y le indica si ha cumplido o no las metas fijadas en la planificación; más aún, advierte el autor, no solo es un proceso para determinar si el rendimiento real es coherente con el rendimiento planificado, y si la organización o unidad de trabajo ha cumplido sus metas. Es también un proceso para analizar las discrepancias entre el rendimiento planificado y el real y así poder modificar los planes y procesos organizativos para satisfacer las necesidades personales y organizacionales.

3. Rol de Mentor: Entre los roles planteados por Quinn (1995), destaca el rol de mentor; éste se enmarca dentro del modelo de relaciones humanas, centrado en los individuos y en los grupos; supone que la participación en la toma de decisiones genera un alto nivel de compromiso. En este rol, según el autor, se espera que el director ayude, sea considerado, abierto, justo con las personas y equipo de trabajo; el líder escucha activamente, apoya las peticiones legítimas, exterioriza el aprecio y ofrece reconocimiento; sugiere entender a los empleados como recursos 
humanos importantes y necesarios que deben ser comprendidos, valorados y desarrollados.

Las tres competencias de este rol son: autocomprensión y comprensión de los demás, comunicación interpersonal y desarrollo de los subordinados.

- Autocomprensión y comprensión de los demás: esta competencia implica que los directivos en su rol de mentor tienen que comprenderse a sí mismos y a los demás, aunque todos los miembros de un equipo de trabajo tienen algo en común, hay que entender a su vez la individualidad de cada persona, el reto consiste, según Quinn (1995), en comprender y valorar los puntos comunes; pero también las diferencias; de igual manera, las múltiples formas en que pueden afectarse las relaciones entre las personas. Conociendo esto, el directivo puede comprender mejor sus propias reacciones ante ellos y las de unos y otros.

- Comunicación interpersonal: Para Quinn (1995) la comunicación interpersonal es quizás "una de las competencias mas importantes y menos comprendida del directivo, pero es vital para desempeñar el rol de mentor; por cuanto la habilidad para comunicarse con eficacia es un activo imprescindible".

La comunicación significa el intercambio de información, hechos, ideas y significado. De igual manera puede utilizarse para coordinar y motivar a las personas. A pesar de las múltiples ventajas que ofrece una comunicación eficaz, no es fácil comunicarse debido, entre otras cosas, a las barreras comunicacionales que pueden generarse en dicho proceso; es más, afirma Quinn (1995).

"Aunque la mayoría de los miembros de las organizaciones piensan que saben comunicarse con eficacia, consideran la comunicación como un importante problema organizativo y ven a los demás como la fuente de problemas. Es muy difícil reconocer y admitir. Los problemas en nuestros propios comportamientos de comunicación".

La carencia de destrezas de comunicación puede generar problemas en los niveles organizativos y de las relaciones interpersonales; las personas comienzan a experimentar conflictos, resistencia al cambio y evitan tener contacto con las otras personas que conforman una unidad o departamento; como consecuencia de esta situación, se dificulta el logro de los objetivos y metas personales y de la propia organización, influyendo en el desarrollo de la moral y la productividad.

- Desarrollo de los subordinados: Mentor significa consejero o guía en quien se confía, un entrenador. Ese guía debe orientar parte de sus esfuerzos del personal mediante procesos de adiestramiento, utilizando los resultados de la evaluación del rendimiento y la información de retorno.

Ruiz (1992) plantea que, el propiciar el desarrollo de los subordinados se constituye en elemento motivador para los empleados, quienes sentirán que sus "jefes" se preocupan por su crecimiento personal y profesional.

4. Rol de facilitador: Éste, a similitud del rol de mentor, se inscribe en el modelo de relaciones humanas. En este rol el directivo fomenta el esfuerzo colec- 
tivo, crea la unión, aumenta la moral del equipo y gestiona los conflictos interpersonales.

Las competencias específicas de este rol son: creación de equipos, toma de decisiones participativas y gestión de conflictos.

- Creación de equipos: en los equipos de trabajo se requiere interacción y coordinación por parte de un líder, sus miembros tienen roles específicos y muy especializados.

- Es relevante, para el funcionamiento eficaz de la organización, que el directivo en concordancia con el puesto de trabajo defina con claridad los roles, evitando la ambigüedad y el conflicto entre los empleados o subordinados.

- Toma de decisiones participativa: para Quinn (1995) ésta no es una técnica única que puede aplicarse universalmente a cualquier situación; sin embargo, es conocido por los jefes las ventajas de involucrar a los empleados en la toma de decisiones; una de esas ventajas está referida al involucramiento y sentido de responsabilidad adquirida cuando forma parte de las decisiones tomadas; además, las discusiones en grupo generan una gama amplia de valores y perspectivas, que representan a su vez la variedad de temas e intereses que están en juego en la decisión.

- Gestión del conflicto. La palabra conflicto comenta Quinn (1995) conlleva connotaciones negativas; en situaciones laborales muchos intentan evitar el conflicto por cuanto genera sentimientos negativos y con ello, un clima de trabajo también negativo.
Existen tres perspectivas del conflicto, una primera, atendiendo a Robbins (2002), según la cual lo ideal en la gestión del conflicto es la eliminación de la fuente de conflicto. Una segunda perspectiva a juicio de Quinn (1995) es la conductual o de relaciones humanas, la cual considera inevitable el conflicto, teniendo en cuenta las diferencias entre las personalidades, las necesidades, metas y valores individuales; la estrategia para la gestión del conflicto se centra en reconocerlo cuando aflora e intentar resolver los temas causantes del conflicto inicial. La tercera, también llamada perspectiva interaccionista; difiere de las anteriores. Robbins (2000) reconoce que, el conflicto no sólo es inevitable, sino que también en ocasiones, es necesario fomentarlo, con el fin de permitir que afloren nuevas ideas $y$ crear fuerzas positivas, generadoras de innovaciones y cambios.

Quinn (1995) plantea que, desde el punto de vista de la tercera perspectiva del conflicto, éste se desarrolla por variados motivos, tales como diferencias en los valores, en las actitudes, en las necesidades o en las percepciones. También se desarrolla entre los individuos cuando surgen malas interpretaciones o errores de comunicación.

El directivo en su rol de facilitador deberá gestionara el conflicto en función de las estrategias y perspectiva que asuma; es decir, las estrategias de no enfrentamiento se encuentran asociadas con las técnicas de evasión y de conciliación, las de control están asociadas con la técnica de competencia y las orientadas a la solución con las de colaboración y compromiso. 
5. Rol de innovador: Este rol forma parte del modelo de sistemas abiertos, responde a la capacidad que debe tener el directivo para adaptarse y darle respuesta al entorno externo. Implica el uso de la creatividad, la gestión de los cambios y de las transiciones organizativas; ofrece la oportunidad de afirmar el valor individual de los empleados dentro de la organización.

Quinn (1995) establece tres competencias para este rol: convivir con el cambio, el pensamiento creativo, y la gestión del cambio. Señala que estas competencias exigen que el mando sea flexible y esté abierto a las nuevas ideas y líneas de pensamiento, así como a nuevos retos que afronte en su rol de directivo.

- Convivir con el cambio: Sostiene el autor antes citado que uno de los retos que debe afrontar el directivo es convivir con los cambios no planificados y, en ocasiones, no deseados; es difícil ajustarse y enfrentar cambios planificados mucho mas cuando estos ocurren y no se está preparado para ello. En ambos casos, se quiere modificar la actitud frente al cambio y un esfuerzo consciente para eliminar la resistencia psicológica al cambio.

- Pensamiento creativo: existe una amplia variedad de comportamientos $y$ características de personalidad asociadas con la habilidad creativa y, lo más importante a juicio de Quinn (1995) el pensamiento creativo es una destreza que cualquier persona puede desarrollar, la idea de la creatividad consiste en el proceso de asociar las cosas o ideas conocidas para crear nuevas combinaciones y relaciones.
En este orden de ideas, autores como Ruiz (1992) y Vera (2002) coinciden en afirmar que, el directivo debe poner a prueba su capacidad creativa dentro de los planteles y de la sociedad en general; aumentar progresivamente el reconocimiento de la necesidad de utilizar las destrezas de pensamiento creativo y critico para confrontar las innovaciones que representan los proyectos educativos.

Para efectos de este trabajo, se seleccionaron los cinco (5), roles antes planteados, como son los de director, coordinador, facilitador, innovador y mentor, en cuanto a que estos tienen aplicabilidad en el contexto de las organizaciones educativas y, sobre todo, en las escuelas básicas de primera y segunda etapa.

\section{Aspectos Metodológicos}

La investigación se clasificó de tipo descriptiva, ya que el objetivo general fue determinar las competencias que posee el gerente educativo de la primera y segunda etapa de la educación básica del Municipio Maracaibo; en este sentido, se buscó identificar y caracterizar las competencias personales y gerenciales de directores y subdirectores, resaltando las cualidades y características de éstas.

De igual manera, se trata de un estudio de campo, en razón de que se desarrolló dentro de las instalaciones de las escuelas seleccionadas, permitiendo obtener la información requerida a través de los propios involucrados; es decir, directores y subdirectores.

La población en estudio estuvo conformada por sujetos; específicamente, por diez (10) directores y dieciocho 
(18) subdirectores para un total de veintiocho (28) gerentes educativos que se desempeñan en las escuelas de primera y segunda etapa de Educación Básica, pertenecientes al Municipio Maracaibo (ver Tabla 1).

Para recabar la información se aplicó un cuestionario estructurado en sesenta y cuatro (64) ítems con alternativas de respuesta: siempre, muy frecuentemente, frecuentemente, pocas veces y nunca. El mismo fue validado a través del juicio de ocho (8) expertos en educación y gerencia; la confiabilidad $(0.88)$ se obtuvo mediante la aplicación del coeficiente de Alpha Cronbach, lo cual indica una alta confiabilidad.

El análisis de la información se realizó utilizando estadística descriptiva, especificamente medidas de tendencia central como frecuencias y promedio de los indicadores. Los resultados se interpretan de acuerdo a un baremo de con- trastación diseñado para tal fin (Tabla 2), en función de la operacionalización de las variables (Tabla 3 ).

\section{Resultados de la investigación}

En este aparte se presentan y analizan los resultados derivados de la aplicación del cuestionario a los directores y subdirectores encuestados. A tal efecto se muestran en la Tabla 4, los datos obtenidos para la variable competencias personales del gerente educativo y los indicadores conocimiento de sí mismo, autorrealización y autocontrol, cuyos valores para los dos primeros, en atención al baremo de interpretación, se ubican en la categoría muy alto dominio de la competencia con un promedio de 3.50 y 3.70 respectivamente. De igual manera, el indicador autocontrol aun cuando se interpreta como alto dominio de la competen-

\section{Tabla 1}

Distribución de la población por escuela

Escuela

Escuela Básica Rafael Escándela

Escuela Básica Nacional 23 de Marzo

Escuela Básica Nacional Carlos Luís Andrade

Escuela Básica Nacional Dr. Marcial Hernández

Escuela Básica Rafael Tomás Jiménez

Escuela Básica Nacional Ángel Álvarez Domínguez Directores

Subdirectores

Escuela Básica Nacional Jorge Washington

Escuela Básica Armando de Jesús Bravo

Escuela Básica Nacional Dr. José María Vargas

Grupo Escolar Ramón Reinoso Núñez

$\begin{array}{cc}1 & 2 \\ 1 & 2 \\ 1 & 2 \\ 1 & 2 \\ 1 & 1 \\ 1 & 1 \\ 1 & 2 \\ 1 & 2 \\ 1 & 2 \\ 1 & 2 \\ 10 & 18\end{array}$

Fuente: Información recabada en las escuelas básicas. Año escolar 2005-2006.

2

2

2

2

1

1

2

2

2

2

18 
Competencias personales y gerenciales de los Directores y Subdirectores... Marcano, Noraida y Finol de Franco, Mineira

\section{Tabla 2 \\ Baremo de interpretación}

\begin{tabular}{lcc}
\hline & \multicolumn{2}{c}{ BAREMO } \\
\hline Alternativas & Intervalo & Categoría de análisis \\
\hline Siempre & $3.41-4.00$ & Muy alto dominio de la competencia \\
Muy frecuentemente & $2.81-3.40$ & Alto dominio de la competencia \\
Frecuentemente & $2.21-2.80$ & Moderado dominio de la competencia \\
Pocas veces & $1.61-2.20$ & Bajo dominio de la competencia \\
Nunca & $1.00-1.60$ & Muy bajo dominio de la competencia \\
\hline
\end{tabular}

Fuente: Finol y Marcano (2005).

Tabla 3

Operacionalización de la investigación

\begin{tabular}{|c|c|c|c|}
\hline \multicolumn{4}{|c|}{ Competencias del Gerente Educativo } \\
\hline Variables & Dimensiones & Indicadores & Items \\
\hline \multirow{10}{*}{$\begin{array}{l}\text { Competencias } \\
\text { personales }\end{array}$} & & Conocimiento de sí mismo & $1,2,3,4$ \\
\hline & & Autorrealización & $5,6,7,8$ \\
\hline & & Autocontrol & $9,10,11,12$ \\
\hline & Rol de Director & Toma de iniciativa & $13,14,15,16$ \\
\hline & & Fijación de metas & $17,18,19,20$ \\
\hline & & Delegación eficaz & $21,22,23,24$ \\
\hline & Rol de Coordinador & Planificación & $25,26,27,28,29$ \\
\hline & & Organización & $30,31,32,33,34$ \\
\hline & & Control & $35,36,37,38,39$ \\
\hline & Rol de Mentor & Comunicación interpersonal & $40,41,42,43$ \\
\hline \multirow{6}{*}{$\begin{array}{l}\text { Competencias } \\
\text { Gerenciales }\end{array}$} & & Desarrollo de los subordinados & $44,45,46,47$ \\
\hline & Rol de Facilitador & Creación de equipos & $48,49,50,51$ \\
\hline & & Toma de decisiones participativas & $52,53,54,55$ \\
\hline & & Gestión del conflicto & $56,57,58,59$ \\
\hline & Rol de innovador & Convivir con el cambio & 60,61 \\
\hline & & Pensamiento creativo & $62,63,64$ \\
\hline
\end{tabular}

Fuente: Finol y Marcano (2005). 


\section{Tabla 4}

Competencias personales del gerente educativo

\begin{tabular}{lcl}
\hline Indicadores & Media & Valoración Cualitativa \\
\hline Conocimiento de sí mismo & 3.50 & Muy alto dominio de la competencia \\
Autorrealización & 3.70 & Muy alto dominio de la competencia \\
Autocontrol & 3.32 & Alto dominio de la competencia \\
Promedio total & 3.50 & Muy alto dominio de la competencia \\
\hline
\end{tabular}

Fuente: Cuestionario aplicado a directores y subdirectores de las escuelas básicas. 2006.

cia, tuvo valores inferiores a los indicadores anteriores con un promedio de 3.32 . Sin embargo, el promedio total (3.50) señala que, en general los directores y subdirectores de las escuelas básicas poseen un muy alto dominio de las competencias personales requeridas para dirigir la institución educativa hacia procesos de excelencia y calidad.

Los resultados anteriores permiten afirmar, que los gerentes de la I y II etapas de las escuelas básicas se apoyan en sus vivencias y características personales para dirigir las organizaciones a su cargo, lo que implica, siguiendo a Senge (1997), que los directores y subdirectores tienen metas intrínsecas; visión personal y profesional sustentada en la autoevaluación como estrategia que les permite autoconocerse desde lo interno para vencer los obstáculos que día a día se presentan en el ejercicio de sus cargos.

Según se observa en los resultados, el personal directivo de las instituciones de educación básica está desarrollando competencias de suma importancia para su desarrollo personal y profesional. Esas cualidades o características le imprimen el dominio suficiente para guiar y orientar a su personal en la búsqueda de soluciones a los problemas que puedan presentárseles; además, les permite coadyuvar a la consecución de los objetivos y metas de los centros educativos. Se confirman así, los planteamientos de Requeijo y Lugo (1997), quienes plantean que una gerencia adecuada es la que logra conjugar personas, distribuir tareas y supervisar trabajo, con el objeto de que produzca bien, esto está asociado con una serie de cualidades personales. La gerencia entendida de esta manera, se soporta en principios de desarrollo social, humano, integral y comunitario, con funciones de integración social y corresponsabilidad del director y subdirector.

Por otra parte, el gerente educativo debe conocerse a sí mismo, implica que tiene deseos, aspiraciones y motivaciones claramente definidos, reconoce sus capacidades, habilidades e identifica sus límites al actuar. Los valores obtenidos para este indicador evidencian muy alto dominio de la competencia en los sujetos encuestados.

Los valores alcanzados por el indicador autorrealización, ponen de manifiesto un muy alto dominio de esta competencia asociada al componente personal; es cuestión relevante para el funcionamiento de las escuelas en estudio, ya que la autorrealización, según Senge (1997) se fundamenta en la adquisición de los propios ideales, de hacer realidad 
las visiones; más aún, Acosta (1998) refiere que es la "aspiración para sentirnos mejores, para buscar la excelencia en lo que se realiza" (p.16).

Los resultados informan que, según la opinión de los directores y subdirectores de las instituciones educativas en estudio, el personal que allí labora y sobre todo los estudiantes, cuentan con gerentes educativos con proyectos de vida personal concretos, con una visión de futuro en base a esfuerzos y logros, lo cual pudiera, de alguna manera, reflejarse en un desarrollo proactivo hacia la excelencia académica al contar la escuela con gerentes que tienen voluntad propia en pro de la búsqueda permanente de la excelencia.

En relación al indicador autocontrol, los encuestados informan que tienen un alto dominio de esa competencia, aunque, en valores promedios inferiores a los indicadores anteriores. Se infiere de los resultados que los gerentes educativos tienen un componente personal fortalecido y que son capaces de interconectar sus aspiraciones y logros, manejar adecuadamente cualquier situación individual, personal y grupal y mostrar un equilibrio en su inteligencia emocional.

En correspondencia con los resultados expuestos para los indicadores analizados, se tiene que, el gerente educativo, en la figura de directores y subdirectores, cuenta con muy alto y alto dominio de las competencias del componente personal, lo cual es trascendental para las escuelas básicas en estudio, por cuanto directores y subdirectores tienen presente su propia voluntad, aspiraciones, motivaciones, percepciones que le permitirán satisfacer sus necesidades superiores de autorrealización.

El Cuadro 1 destaca que, para la variable competencias gerenciales, en la dimensión rol del director con sus respectivos indicadores, sus promedios se ubican en $3.14,3.22$ respectivamente, lo cual puede interpretarse como alto dominio de la competencia. Esta dimensión expresa, según la información obtenida, un alto dominio de las competencias específicas; vale decir, toma de iniciativa, fijación de metas, delegación eficaz, las cuales, en conjunto, definen el rol de director. Estos datos corroboran lo expuesto por Quinn (1995) quien explica que un auténtico gerente debe clarificar las expectativas personales y las de su equipo, fijar metas, ser un iniciador decidido, definir problemas, especificar roles y tareas, entre otros; las tres competencias identificadas por el autor, utilizadas en conjunto constituyen las actividades básicas de la gestión y la organización, visión clara, desafiante y realista. Destaca la toma de iniciativa como competencia clave para la gerencia de los directores y subdirectores de los centros educativos seleccionados, por cuanto éstos como gerentes deben asumir el mando con frecuencia y deben tomar las iniciativas para la ejecución de tareas importantes dentro de las escuelas.

Para la dimensión rol de coordinador, los resultados muestran un promedio de 3.44 (planificación), 3.42 (organización), y 3.07 (control) para los indicadores considerados, lo cual permite afirmar que los directores y subdirectores manejan este rol con muy alto dominio de sus competencias específicas asociadas. Esas competencias, subraya 


\section{Cuadro 1 \\ Competencias gerenciales de los directores y subdirectores de la I y II etapas de las escuelas básicas}

\begin{tabular}{llcl}
\hline & \multicolumn{2}{c}{ Variable Competencias Gerenciales } \\
\hline Dimensiones & Indicador & X & \multicolumn{1}{c}{ Valoración Cualitativa } \\
\hline Rol de Director & Toma de iniciativa & 3.14 & Alto dominio de la competencia \\
& Fijación de metas & 3.32 & Alto dominio de la competencia \\
& Delegación eficaz & 3.19 & Alto dominio de la competencia \\
Rol de & Planificación & 3.44 & Muy alto dominio \\
Coordinador & & & de la competencia \\
& Organización & 3.22 & Alto dominio de la competencia \\
& Control & 3.07 & Alto dominio de la competencia \\
Rol de Mentor & Comunicación interpersonal & 2.98 & Alto dominio de la competencia \\
& Desarrollo de los subordinados & 3.22 & Alto dominio de la competencia \\
Rol de Facilitador & Creación de equipos & 3.07 & Alto dominio de la competencia \\
& Toma de decisiones & 3.22 & Alto dominio de la competencia \\
& Gestión del conflicto & 3.07 & Alto dominio de la competencia \\
& Convivir con el cambio & 1.66 & Bajo dominio de la competencia \\
Rol de Innovador & Pensamiento creativo & 1.77 & Bajo dominio de la competencia \\
& Media total de los indicadores & 2.94 & Alto dominio de la competencia \\
\hline
\end{tabular}

Fuente: Cuestionario aplicado a directores y subdirectores de las escuelas básicas. 2006.

Quinn (1995), constituyen la piedra angular de la gerencia de cualquier centro educativo en relación a que el gerente debe consolidar la planificación anual estratégica y la planificación operativa; con base a estas, seguirá los demás procesos organizacionales-administrativos. En este sentido, llama poderosamente la atención que los directores y subdirectores seleccionaran moderadamente la alternativa frecuentemente, lo que implica que, aunque los resultados señalan un alto dominio de las competencias específicas, aun persisten algunas debilidades en el ejercicio del rol, o que no se realizan con alto nivel de eficiencia, algunas actividades implícitas en él.
Continuando con el análisis se observa que, para la dimensión rol de mentor, los indicadores comunicación interpersonal (2.98) y desarrollo de los subordinados (3.22) se ubican en las categorías alto y muy alto dominio de las competencias. Siguiendo con lo expuesto por Quinn (1995) en relación a este rol, los informantes presentan un comportamiento propio del modelo de relaciones humanas, centrados en los individuos y su equipo, basado en la participación para la toma de decisiones (3.22, muy alto dominio de la competencia), lo cual genera compromiso entre quienes participan.

Quinn (1995), considera que este rol permite describir a los directores como 
justos con las personas y su equipo, escuchan sus peticiones, exteriorizan el aprecio y ofrecen reconocimiento al personal; cuestión que resulta altamente gratificante para los subordinados al contar con gerentes con este cúmulo de cualidades gerenciales.

En relación al rol de facilitador y sus competencias específicas asociadas, se observa que los informantes dicen tener un alto dominio en relación a la creación de equipos (3.07), toma de decisiones (3.22) y gestión de conflictos (3.07). Estas competencias constituyen aspectos fundamentales para el logro de una gerencia participativa, basada en equipos proactivos, identificados con las instituciones donde laboran.

En este sentido, Quinn (1995) menciona que, una de las grandes ventajas de la toma de decisiones participativa está referida al involucramiento y sentido de responsabilidad adquirida cuando se forma parte de las decisiones, además, las discusiones en grupo generan una amplia gama de valores y perspectivas, que representan a su vez, la variedad de temas e intereses que están en juego en la decisión.

Unido a esta decisión de tipo participativa y, en concordancia con una de las competencias específicas, gestión de conflictos, se pudiera interpretar que, al trabajar en equipo, con decisiones participativas, los conflictos presentes en las escuelas en estudio se manejan y enfrentan con mayor facilidad al contar con equipos sólidos.

Para finalizar con el análisis de los cinco roles seleccionados para esta investigación, destacan los datos derivados de las respuestas de los informantes para el rol de innovador, el cual atiende a los indicadores (competencias específicas) convivir con el cambio y pensamiento creativo; con un promedio por indicador de 1.66 y 1.77, respectivamente. Esos resultados señalan un bajo dominio de las competencias asociadas con este rol, lo cual permite afirmar que los gerentes educativos de las escuelas básicas tienen escasa capacidad para adaptarse y darle respuesta al entorno externo, gestionar el cambio y la innovación, usar la creatividad que toda persona tiene y debe aplicar para el diseño e implementación de los proyectos educativos comunitarios, de plantel, entre otros, actividad ésta que requiere de la capacidad y habilidad creativa de todo el personal de las escuelas, pero, sobremanera de los gerentes, ya que ellos deben motorizarlas.

Los resultados arrojados para estos indicadores por la aplicación del cuestionario a los 28 gerentes, no coinciden con los planteamientos de Quinn (1995), quien afirma que el pensamiento creativo es una destreza que cualquier persona puede desarrollar; la idea de la creatividad consiste en el proceso de asociar las cosas o ideas conocidas para crear nuevas combinaciones y relaciones, pero que en el caso de esta investigación no ocurre.

\section{Conclusiones}

Con base en los objetivos propuestos y los resultados, se concluye que:

- Los directores y subdirectores (gerentes educativos) de las escuelas de primera y segunda etapas de educación básica, objeto de estudio, cuentan con las competencias personales, demostrado por los valores muy altos 
obtenidos para los indicadores conocimiento de sí mismo, autorrealización y autocontrol, que la ubican en la categoría de muy alto dominio de las competencias. En este sentido, se podría afirmar que la gestión en los centros educativos se apoya en los componentes personales de quienes direccionan como factores claves para llevar a cabo las acciones organizacionales.

- En relación a la variable competencias gerenciales en los roles de director, coordinador, mentor y facilitador, con sus respectivos indicadores, los resultados permitieron ubicarlos en la categoría muy alto dominio de la competencia; esto pone en evidencia que, según su propia percepción, los directores y subdirectores de las escuelas básicas de I y II etapas, poseen las características y cualidades requeridas para la conducción de las escuelas que gerencian.

- En cuanto al rol de innovador con los indicadores convivir con el cambio (1.66) y pensamiento creativo (1.77), los resultados se corresponden con la categoría muy bajo dominio de la competencia, lo cual llama a la reflexión por cuanto actualmente las escuelas se encuentran en proceso de reformas y de aplicación de innovaciones que requieren de un gerente proactivo, abierto a los cambios y a la ejecución de nuevas acciones e ideas.

En consecuencia, cabe resaltar la necesidad de conjugar la calidad humana a través de las competencias personales del gerente educativo con las cualidades gerenciales para que al unísono proyec- ten en sí mismos y en el entorno, los conocimientos, habilidades, destrezas, sus capacidades para orientar, guiar, planificar, organizar, controlar y liderar su acción gerencial en pro de la calidad educativa de las escuelas básicas seleccionadas y que sirvieron de espacio para el desarrollo de esta investigación.

\section{Referencias Bibliográficas}

Acosta, Manuel (1998). Un proyecto de vida para directores. Planeación de vida y carrera de trabajo. Segunda edición. México.

Álvarez, Manuel (1999). El equipo directivo: Recursos técnicos de gestión. Caracas-Venezuela: Editorial Laboratorio educativo.

Bonilla, Freddy (2001). La gerencia de productividad como alternativa para optimizar la función directiva de las unidades educativas oficiales. Trabajo de Grado. Maracaibo-Venezuela. Universidad del Zulia: Trabajo no publicado.

Carrasco, Milexis (2003). Competencias presentes y requeridas por funciones gerenciales ante nuevas realidades empresariales. Tesis doctoral. Universidad dr. Rafael Belloso Chacín. Maracaibo-Venezuela.

Dalziel, M. Cubeiro, J. y Fernández, G. (1996). Las competencias: clave para una gestión integrada de los recursos humanos. Hay Gruup. España: Ediciones Deusto.

Mercader, Víctor (1998). Gerencia de la vida. Caracas-Venezuela: Editorial TORVIC.

Ministerio de Educación (1997). Currículo Básico Nacional. Mimeografiado. Caracas-Venezuela.

Ministerio de Educación y Deportes (2005). Orientaciones para la construcción 
del proyecto educativo integral comunitario (PEIC). Material mimeografiado. Caracas-Venezuela.

Ministerio de Educación, Cultura y Deportes (2000). Proyecto Educativo Nacional. Caracas-Venezuela: Material Mimeografiado.

Ministerio de Educación, Cultura y Deportes (2003). Nuevo Currículo Básico Nacional. Caracas-Venezuela: Material mimeografiado. Tercera versión. Sistematizado por la Socióloga Marisela Morales. Maracaibo-Venezuela. Zona educativa Zulia.

Quinn, Robert (1995). Maestría en Gestión de las Organizaciones. Traducción, Carmen León Pérez. Madrid-España: Ediciones Díaz Santos.

Robbins, S. (2002). Comportamiento organizacional. México: editorial Mc Graw Hill.

Ruiz, José (1992). Gerencia en el aula. Caracas-Venezuela: Editorial Laboratorios educativos.
Senge, Peter (1997). La Quinta Disciplina. Cómo impulsar el aprendizaje en la organización inteligente. BarcelonaEspaña: editorial Juan Graniza S.A.

Silíceo Aguilar, Alfonso (1997). Líderes para el siglo XXI. México: editorial Mc Graw Hill.

Vásquez, Minerva (2005). Perfil basado en competencias gerenciales de los coordinadores administrativos en las instituciones de educación superior. Tesis Doctoral. Universidad Dr. Rafael Belloso. Chacín Maracaibo-Venezuela.

Vera, Carlos (2002). Perfil de competencias del líder en proyectos de investigación y desarrollo del sector público. Tesis Doctoral. Universidad Dr. Rafael Belloso Chacín. Maracaibo-Venezuela. 